\title{
Zur Verbreitung des Milzbrandes in Württemberg.
}

Von

\author{
H. Beisswaenger, \\ Veterinär-Assessor in Stuttgart.

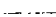 \\ (IIierzu TaP. IV u. V.)
}

Im Anschluss an die Mittheilungen des Hrn. Medicinalrath Dr. Rembold über einzelne Fälle einer Einschleppung des Milzbrandes durch sogenannte Wildhäute ${ }^{1}$ möchte ich zwei kartographische Uebersichten, welche ein Bild von der allgemeinen Verbreitung des Milzbrandes in Württemberg und von dem Umfang des gesammten Wildhautgerbereibetriebes bezw. der räumlichen Vertheilung der Wildhautgerbereien im Lande geben, zur Kenntniss weiterer Kreise bringen, da diese Uebersichten einigen Aufschluss über die Verbreitungsweise des Milzbrandes in Württemberg gewähren und vielleicht auch ein allgemeineres Interesse bieten.

Der Milzbrand hat, wie aus den vom Gesundheitsamt veröffentlichten Jahresberichten über die Verbreitung der Thierseuchen im Deutschen Reiche des Näheren ersehen werden kann, in Württemberg in den letzten Jahren eine auffallend grosse Zahl von Thieren befallen; die Zahl der Milzbrandfälle war früher wesentlich geringer, sie hat in den letzten Jahren stetig zugenommen. Wenn diese stetige Vermehrung der zur amtlichen Kenntniss gelangten Zahl der Milzbrandfälle zum Theil auch als eine Wirkung der im Jahre 1885 eingeführten Entschädigungsleistung für an Milzbrand gefallene Thiere zu betrachten sein dürfte, insofern früher vielleicht verschiedene Seuchenausbrüche verheimlicht wurden, seit Gewährung einer Entschädigung aber alle Seuchenfälle angezeigt werden, so glaube ich unter den gegebenen Verhältnissen doch annehmen zu

1 Diese Zeitschrift. Bd. IV. S. 498 ff. und Bd. V. S. $506 \mathrm{ft}$. 
müssen, dass es sich in den letzten Jahren um einen thatsächlichen Zuwachs an Kilzbrandfällen handelte und dass Verheimlichungen doch nur verhältnissmässig selten vorkamen.

Eine Erklärung für dieses häufige Vorkommen bezw. für die stetige Zunahme des Milzbrandes konnte seither nach Lage der Verhältnisse aber nicht gefunden werden.

Als Folge früherer unzweckmässiger Beseitigung von Milzbrandcadavern oder von Theilen solcher Cadaver kann nur ein verhältnissmässig kleiner Theil der vorgekommenen Fälle angesehen werden. In den oberamtsthierärztlichen Jahresberichten pro 1887, in welchem Jihre diese Berichte erstmals auch in Absicht auf die Seuchenätiologie in ausführlicher Weise bearbeitet worden sind, werden nur in wenigen Gemeinden rlie aufgetretenen Milzbrandfälle als muthmasslich auf die genannte Weise entstanden erklärt; nach diesen Berichten sind höchstens die in den Gemeinden Dusslingen 0./A. Tüibingen, Hardt 0./A, Oberndorf, Gürtringen 0./A. Herrenberg (?), sowie in einigen Gemeinden des Oberamts Ellwangen vorgekommenen Fälle auf eine in der gedachten Weise zu Stande gekommene Ansiedelung von Milzbrandkeimen an bestimmten Oertlichkeiten zurückzuführen. In allen anderen betroffenen Gemeinden des Landes liegt aber absolut kein Grund zu der Annahme einer solchen Verbreitungsweise des Milzbrandes vor; an eine Verbreitung der Seuche von den üffentlichen Verscharrungsplätzen aus ist bei den bestehenden Einrichtungen in den meisten Orten ebenfalls nicht wohl zu denken.

Ferner sind nur verhältnissmässig wenige Seuchenfälle bekannt geworden, welche durch andere gleichzeitige Seuchenausbrüche veranlasst wurden, sei es durch mittelbare oder unmittelbare Uebertragung von Thier auf Thier, durch unerlaubte Verwendung von Cadavern oder von Theilen derselben u. dergl. m. Hier sei auch bemerkt, dass der Milzbrand bei Sichafen, welcher anderwärts so vielfach zur Verschleppung der Seuche beizutragen scheint, bei uns fast ganz unbekannt ist.

Durch mangelhafte Desinfection verseuchter Räumlichkeiten können ebenfalls nur wenige Milzbrandfälle entstanden sein, da wiederholte Seuchenausbrüche in früher betroffenen Gehöften nur verhältnissmässig selten beobachtet wurden.

Bei der Mehrzahl der vorgekommenen Seuchenfälle war vielmehr die Infectionsweise überhaupt nicht zu ermitteln, es blieben bei dem Fehlen aller Anhaltspunkte für die Ableitung von früheren oder gleichzeitigen Seuchenausbrüchen und bei dem Mangel jeglicher nachweisbaren Infeetionsgelegenheit die meisten Milzbrandfälle ätiologiseh vollkommen räthselhaft. Unter diesen Umständen war eben an eine häufige Neueinschleppung nicht näher bekannter Art zu denken. 
Als es nun gelungen war, den directen Nachweis zu liefern, dass Iunter den aus überseeischen Ländern eingeführien Rinderhäuten, den sogenannten Wildhäuten, sich hin und wieder solche Häute befinden, welche keimfähige Milzbrandsporen an sich tragen, und als erhoben war, dass verschiedene Sezchenausbrüche offenbar durch diese Häute veranlasst worden waren, als demnach eine neue Infeetionsquelle ermittelt war, schien tuun Licht in das über der Verbreitungsweise des Milzbrandes in Württemberg schwebende Dunkel kommen zu wollen.

Die positiven Ergebnisse der in den eingangs erwähnten Veröffentlichungen mitgetheilten Erhebungen und Untersuchungen gaben daher Anlass zur näheren Prüfung der Frage, ob und inwieweit der Einfuhr und der Verarbeitung der sogenannten Wildhäute im Allgemeinen ein Einfluss wuf die Verbreitung des Milzbrandes im Lande zuzumessen sei.

$\mathrm{Zu}$ diesem Zweck wurden zunächst theils amtliche, theils private $\mathrm{Er}$ hebungen angestellt und zwar über die Einfuhr und Verarbeitung von sogenannten Wildhäuten einerseits und über die Verbreitung des Milzbrandes innerhalb der betroffenen Bezirke andererseits.

Die alljährliche Einfuhr von Wildhäuten ist naç diesen Erhebungen eine ganz bedeutende. Die Wildhautgerberei wird im Lande in grossem Umfange betrieben und bildet in verschiedenen Gemeinden sogar das vorherrschende Gewerbe. In diesen Gemeinden befinden sich neben den Gerbereien noch grosse, jüdischen Händlern gehörige Hautlager, in welche grosse Mengen von Häuten eingebracht werden und von wo aus die Häute dann über das ganze Land versandt werden. In geringerer Zahl befinden sich Wildhautgerbereien in sehr vielen Gemeinden und rereinzelte Wildhautgerbereibetriebe sind über das ganze Land zerstreut.

Die Wildhautgerberei liegt in der Hauptsache in den Händen Kleingewerbetreibender, welche vielfach noch auf einen Oekonomiebetrieb und auf Viehhaltung angewiesen sind.

Die Gerbereigehöfte liegen aus erklärlichen Gründen in der Regel an fliessendem Wasser; die Abläufe aus den Geschäftslocalen werden meistens direct in die Flüsse oder Bäche eingeleitet. Auch ist es allgemein gebräuchlich, die Häute vor der Verarbeitung zum Zwecke des Einweichens längere Zeit in das fliessende Wasser einzulegen.

Nach dem Einweichen werden die nassen Häute dann in der Regel in gut bedeckten Gruben dicht zusammengehängt, um die Haare zum Ausfallen zu bringen. Die Thierhaare und der beim Abschaben der aus den Gruben genommenen Häute sich ergebende Abfall finden sodann als beliebtes Düngemittel vielfach Verwendung.

Wenn man nun bedenkt, dass in Württemberg alljährlich eine grosse Menge sogenannter Wildhäute eingeführt wird und dass unter diesen 
Massen sich öfters Häute mit Milzbrandkeimen befinden werden, sowie dass bei der Art des Gerbereibetriebes eine Verschleppung etwa vorhandener Sporen in mannigfacher Weise geschehen kann, so erscheint es von vornherein naheliegend, dass in der Wildhautgerberei eine grosse Gefahr der Seucheneinschleppung gegeben ist. Einerseits können etwa vorhandene Milzbrandsporen auf dem Transport der Häute, sowie in den Gerbereigehöften und Lagerräumen mit dem abfallenden Hautstaube ausgesät und auf alle möglichen Weisen weiter verbreitet werden, andererseits können die Sporen auch bei der Verarbeitung der Häute Verbreitung finden, sie können sodann mit den aus den Geschäftslocalen stammenden Abläufen oder durch das übliche Einlegen der Häute in's fliessende Wasser auch in öffentliche Wasserläufe gelangen, dort weiter geschwemmt und möglicher Weise bęi Ueberschwemmungen mit dem Flussschlamm auf Aecker und Wiesen hinausgetragen werden, und endlich ist eine Verbreitung der Sporen durch die Benutzung des sogenannten Haardüngers noch möglich. Durch den Wildhautgerbereibetrieb kann demnach nicht bloss das Gerbereigehöft selbst, sondern auch dessen ganze Nachbarschaft, ja durch Vermittelung der öffentlichen Wasserläufe und in Folge der Verwendung des sogenannten Haardüngers können auch weiter entfernt gelegene Gehöfte und Gemeinden gefährdet werden. Ebenso ist schon auf dem Transport der Häute eine Reihe von Verschleppungsmöglichkeiten gegeben.

Selbstverständlich darf aber nicht erwartet werden, dass jeder Wildhautgerbereibetrieb auch Milzbrandausbrüche veranlassen muss, da ja, selbst bei der Einfuhr milzbrandsporentragender Häute, nur unter ganz bestimmten Voraussetzungen eine wirksame Uebertragung zu Stande kommen kann.

Diese allgemeinen Betrachtungen dürften genügen; ein Eingehen anf alle Einzelnheiten, unter welchen eine Uebertragung zu Stande kommen kann, halte ich nicht für geboten.

Ausgehend von diesen Erwägungen habe ich nun zunächst Uebersichtskarten über die Vertheilung der vorhandenen Wildhautgerbereien und über die Verbreitung des Milzbrandes nach Bezirken hergestellt, um zu prüfen, inwieweit die obigen Voraussetzungen im Allgemeinen zutreffen. Um die verschiedenen betroffenen Bezirke unter sich hinsichtlich des Grades ihrer Verseuchung vergleichen zu können, wurde bei diesen Darstellungen für jeden Bezirk die Zahl der auf je 10,000 Stück des Gesammtrindviehbestandes des Bezirkes kommenden Milzbrandfälle beim Rinde zu Grunde gelegt und eine Abstufung in der Verseuchung in der Weise durchgeführt, dass die Bezirke, in welchen auf je 10,000 Rinder bis zu 5.00 Wilzbrandfälle kamen, auf die erste Stufe. diejenigen mit 5.01 bis 10.00 Milzbrandfällen auf die zweite Stufe, die mit 10.01 bis 
20.00 auf die dritte, die mit 20.01-30.00 auf die vierte und die mit 30.01-40.00 Fällen auf je 10,000 Rinder auf die fünfte Stufe gestellt wurden. Für diese Darstellungen wurden zwei Jahrgänge benutzt, es wurden hierza, wie auch für die beigegebenen beiden Gemeindekarten, die Jahrgänge 1887 und 1888 gewählt, da in diesen beiden Jahren sicher alle vorgekommenen Milzbrandfälle auch zur amtlichen Kenntniss gelangt sind.

Aus diesen Uebersichten, welche ihres geringeren Werthes wegen nicht beigegeben sind, liess sich nun ersehen, dass in beiden Jahren eine gewisse Uebereinstimmung in der Verbreitung des Milzbrandes bestand und dass der die meisten Wildhautgerbereien umfassende Schwarzwaldkreis jedesmal auch am stärksten verseucht war, dass der Neckar- und der Jagstkreis, welche etwas weniger Gerbereien enthalten, auch im Grade der Verseuchung in beiden Jahren in zweiter Linie standen und dass endlich der Donaukreis, in welchem die Wildhautgerberei nur verhältnissmässig wenig betrieben wird, in beiden Jahren auch nur unbedeutend verseucht sich zeigte. Innerhalb der drei stärker betroffenen Kreise waren in beiden Jahren namentlich wieder solche Bezirke in hervorragenderem Maasse betroffen, welche entweder selbst viele Wildhautgerbereien enthalten oder welche Flussgebiete umfassen, in welchen stromaufwärts zahlreiche Gerbereien sich befinden.

Um nun die Verhältnisse in den betroffenen Bezirken weiter verfolgen zu können, habe ich in den beifolgenden Karten innerhalb der betroffenen Bezirke die verseuchten Gemeinden markirt, dieselben sind je nach dem Grade ihres Betroffenseins durch verschiedene schwarze Unterstreichung der Gemeindenamen gekennzeichnet. Da die Lage der geschlossenen Ortschaft für die Beurtheilung der vorliegenden Frage weniger in Betracht kommt, als die Ausdehnung der Gemeindemarkung; so wurden die Markungsgrenzen eingezeichnet. $\mathrm{Zu}$ bemerken ist noch, dass in diesen Karten die absolute Zahl der vorgekommenen Milzbrandfälle angegeben ist.

Diejenigen Gemeinden, in denen Wildhautgerbereibetriebe sich befinden, sind durch rothe, der Bedeutung dieser Betriebe nach verschiedene Unterstreichung der Ortsnamen hervorgehoben. Bei diesen Gemeinden kommt die Ausdehnung der Markung in der Regel weniger in Betracht, da die Gerbereien ja auch meistens in der geschlossenen Ortschaft liegen; es ist daher die Lage der Ortschaften selbst durch kleine Kreise angedeutet worden.

In der Karte pro 1888 konnten die Einträge für den Bezirk Waldsee nicht gemacht werden, es ist dies jedoch von keiner Bedeutung, da dort nur wenige Seuchenfälle vorkamen. Endlich ist noch zu erwähnen, 
dass hei der Beurtheilung der aufgeworfenen Frage yon dem Bezirk Mergentheim abzusehen sein dürfte, da Grund zu der Annahme vorliegt, dass es sich dort in vielen Fällen gar nicht um Milzbrand, sondern um eine andere ähnliche Krankheit geaandelt huben wird.

Eine genaue Betrachtung der Karten wird weiter führen als eine umfingreiche Bescbreibung derselben, weshalb ich es unterlasse, auf alle Finzelnheiten einzugehen, und mich darauf beschränke, nur die Verhältilisse in denjenigen Bezirken, welche in den auf S. 182 genannten Karten, also im Vergleich zu dem Gesammtrindviehbestand des Bezirkes, am stärksten verseucht sich zeigten, näher zu hespre:

In dem verhältnissmässig am meisten betroffenen Sthwarzwaldkreise verseuchten in besonders hohem Grade in beiden Jahren die Oberämter Tuttlingen und Balingen (dritte Stufe in den Bezirksharten).

Im Bezirk Tuttlingen ist in beiden Jahren vorzugsweise die Stadt Tuttlingen selbst, eine der bedeutendsten Gerberstïdte des Landes, betroffen worden; im Uebrigen wurden nur noch und zwar in geringerem Grade einige in der nächsten Umgebung von der Oberamtsstadt und einige donauabwärts von derselben gelegene Gemeinden ergriffen. Ueber die specielleren Verhältnisse in der Stadt Tuttlingen giebt die Mittheilung in Band IV dieser Zeitschrift näheren Aufschluss.

Ebenso ist die Seuche im Bezirk Balingen mit wenigen Ausnahmen nur in dem Hauptgerberorte Ebingen aufgetreten. Schon in dem Jahresberichte pro 1884 führt Oberamtsthierarzt Deigendesch-Balingen aus, dass die grösste Zahl der beobachteten Milzbrandfälle in der Stadt Ebingen vorkam und dass meistens Gehöfte von Rothgerbern, welche aus überseeischen Ländern eingeführte Wildhäute verarbeiteten, von der Seuche betroffen wurden. Nach weiteren Mittheilungen Deigendesch's hat der Milzbrand auch seither immer die Ställe der Rothgerbereien und der Umgebung der grossen Hantlager bevorzugt.

Im Bezirk Tübingen, welcher ebenfalls in beiden Jahren, jedoch mässiger (1887 - zweite und 1888 - dritte Stufe der Bezirkskarten) verseuchte, fällt die Hauptzahl der vorgekommenen Seuchenfälle auf die oben S. 180 schon genannte Gemeinde Dusslingen, doch sind im Uebrigen ausser der Stadt Tübingen, in welcher sich eine sehr grosse Wildhantgerberei befindet, und deren nächster Umgebung auch nur wenige Gemeinden betroffen.

Nur in einem Jahre in höherem Madisse verseucht waren die Bezirke Reutlingen (1888 - vierte Stufe), Nürtingen, Calw (1887 je zweite Stufe) und Rottweil (1888 - zweite Stufe).

In der Oberamtsstadt Reutlingen betinden sich bei einem verhältnissmässig kleinen Viehbestand zahlreiche Wildhautgerbereien und auch 
griissere Hautlager. Die Milzbrandfälle vertheilen sich im Bezirk Reutlingen in beiden Jahren in der Hauptsache auf die Stadt Reutlingen und auf die beiden im Echazthale fiussabwärts von Reutlingen gelegenen Gemeinden. Nur im Jahre 1888 kamen ausserdem noch vereinzelte Fälle in an die Oberamtsstadt grenzenden Gemeinden vor. In der am meisten befillenen, im Echazthale gelegenen Gemeinde Betzingen war der Wasserstand nach den Berichten des Oberamtsthierarztes von Mitte Juni des Jahres 1888 an in Folge der anhaltenden Regen ein sehr hoher; ob es in jener Zeit zu einer förmlichen Ueberschwemmung auf der Betzinger Markung gekommen ist, ist aus den Berichten leider nicht bestimmt zu ersehen. Bemerkenswerth ist, dass ca. 80 Procent ron den im Jahre 1888 im Bezirk Reutlingen vorgekommenen Milzbrandfallen in der Zeit von Witte Juni bis December aufgetreten sind, während nur etwa 20 Procent der Seuchenausbrüche auf die Zeit von Januar bis Mitte Juni fallen.

Der Bezirk Nürtingen zeigt in beiden Jahren ebenfalls eine gewisse Uebereinstimmung in der Vertheilung der Senchenfälle. In beiden Jahren wurde die Oberamtsstadt Nürtingen, in welcher ca. 12 Gerber mit der Verarbeitung von Wildhäuten sich befassen, und die flussabwärts von ihr gelegene Gemeinde Oberbvihingen betroffen. Während im Jahre 1888 nur noch ein einzelner Fall in einer Nachbargemeinde vorkam, waren im Jahre 1887 in einer weiter entfernten Gemeinde mehrere Fälle zu verzeichnen. Diese weiter betroffene Gemeinde, Neckartenzlingen, ist aber wieder eine solche, welche ein Flussgebiet (Erms), in dem flussaufwärts zahlreiche Wildhautgerbereien (Mezingen) sich befinden, umfasst.

Im Uebrigen sind im Schwarzwaldkreise, abgesehen von der schon auf S. 180 angeführten Gemeinde Gärtringen 0./A. Herrenberg, keine bedeutenderen Seuchenherde mehr vorhanden, es handelt sich sonst nur noch um wenige, meist vereinzelte Seuchenausbrüche. Aber auch in der Vertheilung dieser Fälle kann bei näherer Betrachtung der Karten das erwähnte Verhältniss zur Lage von Gerbereien mehrfach beobachtet werden.

Im Neckarkreis verseuchten in beiden Jahren in hervorragenderem Maasse die Bezirke Marbach (1887 - dritte und 1888 - fünfte Stufe) und Backnang (1887 - zweite und 1888 - dritte Stufe).

- In dem verhältnissmässig am stärksten verseuchten Bezirke des Landes, in dem Oberamt Marbach, fallen weitaus die meisten Fälle auf die im Murrthale gelegenen Gemeinden, welche beinahe alle vom Milzbrand betroffen wurden. Weiter ist die nach der Einmündung der Murr in den Neckar an dem letzteren gelegene Gemeinde des Bezirkes in beiden Jahren ebenfalls mässig verseucht und im Jahre 1888 sind auch zwei weitere dort liegende Gemeinden des Bezirkes Ludwigsburg 
noch betroffen worden. In den nicht im Murrthale gelegenen Gemeinden des Oberamtes Marbach kamen aber, abgesehen von einer mit ihrer Markung noch in die Neckarebene hineinreichenden Gemeinde, nur noch wenige und vereinzelte Fälle vor. Das Murrthal war nach dem oberamtsthierärztlichen Jahresberichte pro 1887 Ende Yai und Anfangs Juni 1887 überschwemmt; die Seuchenfälle traten erst vom September 1887 an auf. Weiter ist auch in den meisten Fällen ermittelt worden, dass die betroffenen Thiere kurz vor ihrer Erkrankung noch Futter von den überschwemmten Wiesen erhalten hatten. Die ron dem dermaligen Oberamtsthierarzt Hofstadt seither bei jedem vorkommenden Falle angestellten Erhebungen ergaben in der Mehrzahl der Seuchenausbrüche dasselbe. Wildhautgerbereien befinden sich nun zwar in dem Bezirke Marbach nicht, doch ist flussaufwärts an der Murr in nächster Nähe die grösste Gerberstadt des Landes, die Oberamtsstadt Backnang mit über 100 Gerbereien, in denen grosse Mengen sogenannter Wildhäute (jährlich etwa eine Million) verarbeitet werden, gelegen.

Im Bezirke Backnang selbst vertheilen sich die zahlreichen Milzbrandfälle in beiden Jahren, abgesehen von wenigen vereinzelten Fällen, auf die Stadt Backnang und die weitere Gemeinde, in der Wildhäute gegerbt werden. In Backnang sind ausser den Wildhautgerbereien auch grosse Hautlager. Auch Oberamtsthierarat Haefele-Backnang hat nach seinem Jahresberichte pro 1886 die Beobachtung gemacht, dass der Milzhrand hauptsächlich in. Ställen auftrat, welche früher als Wildhautmagazine gedient hatten, und dass die Krankheit auch rorzugsweise die Viehbestände von Gerbern ergriff.

Nur in einem Jahre und zwar im Jahre 1888 verseuchten im Neckarkreis in höherem Grade die Oberämter Böblingen (dritte Stufe) und Neckarsulm (zweite Stufe der Bezirkskarten).

Im Bezirk Böblingen kamen die meisten Fälle im Würmbachthale vor, im Uebrigen wurde nur noch die Oberamtsstadt stärker betroffen. Im Würmbachthale wurden zwar im Jahre 1888 keine Wildhäute mehr verarbeitet, doch geschah dies in den Jahren vorher in dem oberhalb der betroffenen Gemeinde gelegenen Orte. Ueber die detiologie der Milzbrandfälle in der Oberamtsstadt ist nichts bekinnt.

Für den Bezirk Neckarsulm ist erwähnenswerth, dass im Jahre 1888 nur solche Gemeinden betroffen worden sind, welche im Kocher-, Jagst- oder Neckarthale liegen.

Im ganzen Neckarkreis ist ausserdem nur noch eine Gemeinde und zwar Waldenbuch im Amtsoberamt Stuttgart in einem Jahre (1887) in höherem Grade verseucht. Die Seuche ist dort schon seit längerer Zeit 
alljährlich aufgetreten, der erste F'all soll jedoch ebenfalls in einer früheren Gerberei vorgekommen sein.

Weiter sind im Neckarkreis nur noch vereinzelte Fälle bekannt geworden, aber auch diese kamen vielfach im Rayon von Wildhautgerhereien vor.

Aehnliche Verhältnisse bietet auch der Jagstkreis. In beiden Jahren verseuchte dort, abgesehen vou Mergentheim, in hervorragender Weise nur Crailsheim (dritte Stufe der Bezirkskarten). In diesem Bezirk sind in besonderem Maasse die Stadt Crailsheim, in welcher verschiedene Wildhautgerbereien betrieben werden, und die jagstabwärts von Crailsheim gelegenen Gemeinden betroffen worden. Der Milzbrand hat jedoch auch in einigen anderen Orten des Bezirkes, und wie es scheint dauernd Boden gefasst, in Orten, welche ihrer Lage nach von Wildhautgerbereien nicht beeinflusst werden können, es wäre denn, dass in ihnen Hadrdünger zur Verwendung kommt.

Nur in einem Jahre und zwar im Jahre 1888 kamen als stärker verseucht hinzu die Bezirke Gerabronn, Künzelsau, Heidenheim und Ellwangen (sämmtlich zweite Stufe). In Gerabronn und Künzelsau sind verschiedene im Jagstthale unterhalb Crailsheim, wo auch noch einzelne Wildhautgerbereibetriebe eingestreut sind, gelegene Gemeinden betroffen wordeu; ferner sind im Bezirk Gerabronn einzelne Gemeinden in Vorbachthal, wo ebenfalls einzelne Gerbereien sich befinden, und im Bezirk Künzelsau einzelne Gemeinden im Kocherthale verseucht. Weiter ist im Bezirk Heidenheim in beiden Jahren nur die unterhalb der Gerbereien in Giengen an der Brenz gelegene Gemeinde Hermaringen befallen. Auf den Bezirk Ellwangen ist schon auf S. 180 hingewiesen worden.

Im Uebrigen liegen auch in den weniger betroffenen Bezirken, wie die angeschlossenen Karten ergeben, die Verhältnisse vielfach ganz ähnlich.

In dem den grössten Viehstand aufweisenden Donaukreis, in welchem die Wildhautgerberei nur wenig betrieben wird, ist die Zahl der vorgekommenen Milzbrandfälle eine verhältnissmässig geringe. Als interessant ist hier hervorzuheben die Vertheilung der Milzbrandfälle im Bezirk Kirchheim, die stärkere Verseuchung verschiedener an der Donau gelegener Gemeinden und endlich noch die Verbreitung der Seuche im Bezirk Leutkirch.

Aus den beigegebenen kartographischen Uebersichten ist demnach zu ersehen, dass innerhalb der verhältnissmässig am stärksten verseuchten Bezirke namentlich wieder solche Gemeinden in höherem Maasse vom Milzbrand betroffen worden sind, in welchen die Wildhautgerberei in grösserem Umfang betrieben wird, oder solche, welche flussabwärts von Gerberorten liegen; in beiden Jahren zeigten sich anch so ziemlich die- 
selben Gemeinden als am stärksten verseucht. In der Nachbarschaft von Gerberorten waren Milzbrandfälle ebenfalls nicht selten, in Orten aber, welche zu Wildhautgerbereien in gar keiner Beziehung stehen, sind auch, abgesehen von wenigen Ausnahmen, in der Regel nur vereinzelte Fälle vorgekommen.

Bei dieser Sachlage erscheint denn im Hinblick auf das Ergebniss der von Hrn. Medicinalrath Dr. Rembold mitgetheilten Erhebungen und Untersuchungen und bei dem Umstand, dass die alljährliche Einfuhr von sogenannten Wildhäuten eine ganz bedeutende ist und dass bei der Art des Gerbereibetriebes und vielleicht auch der Verwendung der Gerbereiabfälle eine Reihe von Möglichkeiten für die Verschleppung an den Wildhäuten etwa haftender Milzbrandsporen gegeben ist, sowie in Berücksichtigung der von verschiedenen Oberamtsthierärzten gemachten, oben angeführten Beobachtungen die Annahme nicht unbegründet, dass der Wildhautgerberei ein wesentlicher Einfluss auf die Verbreitung des Milzbrandes in Württemberg zukommt und dass in dem umfangreichen Betriebe der Wildhautgerberei die Hauptursache des häutigen Vorkommens des Milzbrandes in Württemberg zu suchen ist.

Da es bei der Natur der Sache wohl überhaupt nicht möglich sein wird, die Ursache der häufigen Milzbrandausbrüche in Württemberg mit absoluter Sicherheit festzustellen, so dürfte dem Ergebniss meiner Erhebungen, das wenigstens einen hohen Grad von Wahrscheinlichkeit für sich hat, doch immerhin ein gewisser Werth beizulegen sein. 